\title{
Antimicrobial Efficiency of Biopure MTAD and Sodium Hypochlorite as Endodontic Irrigants
}

\section{Hayam Y Hassan ${ }^{1 *}$, Maged M Negm ${ }^{2}$, Salah Abdalla ${ }^{3}$ and Ahmad Fawzy ${ }^{4}$}

${ }^{1}$ Associate Professor of Endodontic, Faculty of Dentistry, Suez Canal University, Ismailia, Egypt

${ }^{2}$ Professor of Endodontic, Faculty of Oral and Dental Medicine, Cairo University, Giza, Egypt

${ }^{3}$ Professor of Microbiology and Immunology, Faculty of Pharmacy, Suez Canal University, Ismailia, Egypt

${ }^{4}$ Associate Professor of Operative Dentistry, Faculty of Dentistry, Suez Canal University, Ismailia, Egypt

*Corresponding Author: Hayam Y. Hassan, Associate Professor of Endodontic, Faculty of Dentistry, Suez Canal University, Ismailia, Egypt.

DOI: 10.31080/ASDS.2020.04.0787
Received: January 09, 2020

Published: February 20, 2020

(C) All rights are reserved by Hayam $\mathbf{Y}$

Hassan., et al.

\section{Abstract}

The Aim: This in vitro study is to compare the antimicrobial efficiency of Biopure MTAD and its effect together with sodium hypochlorite and hydrogen peroxide on Enterococcus faecalis.

Methods: One hundred micro-liters of broth inoculated with Enterococcus faecalis was spread on sterile Mueller-Hinton agar (MHA) with sheep blood for each in six sterile glass plates. Approximately fifty micro-liters of each freshly prepared tested material were added onto the filter papers in the prepared plates. The plates were incubated at $37 \circ \mathrm{C}$ for 48 hours and the zones of inhibition were measured. Furthermore, a total of 55 standardized root canals length and diameter were sterilized. Five teeth cultured to act as negative control (-ve). For infection of the root canals, a trypticase soy broth inoculated with Enterococcus faecalis was used. The roots incubated for 48 hours. Five teeth cultured to act as positive control (+ve). The remaining teeth were divided into three groups $\mathrm{M}, \mathrm{C}$ and D ( $\mathrm{n}=15)$. Group M was irrigated with Bio pure MTAD. Groups C and D were irrigated with $(5.25 \%)$ and $(2.5 \%)$ sodium hypochlorite. Two or three sterile paper points were inserted into every root canal, and then transferred aseptically into its markedly corresponding Wassermann tube for each root. All the marked Wassermann tubes were incubated at $37 \circ \mathrm{C}$ for 48 hours then microbial growth of Enterococcus faecalis was verified by turbidity of the broth.

Results: Bio pure MTAD recorded the best results in respect of inhibiting the growth of Enterococcus faecalis. Moreover, additives as (1.3\%) sodium hypochlorite and (20\%) hydrogen peroxide decreased the efficiency of Bio pure MTAD against Enterococcus faecalis. Conclusions: Bio pure MTAD completely inhibited the growth of Enterococcus faecalis. Moreover sodium hypochlorite had poor antimicrobial efficiency against Enterococcus faecalis.

Keywords: Biopure MTAD; Enterococcus faecalis; Antimicrobial Efficiency; Sodium Hypochlorite

\section{Introduction}

The most common causative factor of pulpal disease is microorganisms. This is because the body defending cells and molecules from periarticular tissues do not function in such tissue [1]. Once the pulp is necrotic, only a restricted subset of species can colonize in the pulp space. Some groups of oral microbial species have been reported to be associated with particular forms of periarticular diseases, which confer a semi-condition and cannot reach to micro-organisms location in necrotic pulps specific etiology to endodontic infections [2]. In general, species more frequently found in primary root canal infections usually belong to the genera Bacteroides, Fusobacterium, Prevotella, Porphyromonas, Treponema, Peptostreptococcus, Eubacterium, Actinomyces, and Streptococcus [3].

Enterococcus faecalis is Gram-positive, non-spore forming cocci that occur singly, in pairs, and in short chains. It is a facultative anaerobe and is a normal commensal adapted to the ecologically complex environments of the oral cavity and gastrointestinal and vaginal tracts. Enterococcus faecalis has been occasionally detected in primary root canal infections [4]. It has innate resistance to a variety of antibiotics such as the beta-lactamas, aminoglycosides, 
vancomycin, and trimethoprim-sulfamethoxazole. Searches reported that Enterococcus faecalis can be found in several cases of persistent infections [5,6]. Generally, the disinfecting process is essential for successful root-canal treatment, and irrigation of the root canals to remove microorganisms and cleanse pulpal debris is an important step. There is a great importance of using antimicrobial irrigants during the chemo-mechanical preparations. Effective endodontic irrigants require a broad spectrum of antimicrobial activity, as well as a relative lack of toxicity to periapical and oral mucosal tissues. Root canal irrigation is designed to remove pre-existing debris, dentin chips, bacteria, toxic products, and substrates essential for bacterial growth [7]. Sodium hypochlorite has been the irrigant of choice for nonsurgical endodontic procedures. Sodium hypochlorite dissolves necrotic and vital tissue, has antimicrobial activity, and aids as a lubricant in the canal. It also has least toxicity when kept inside the limits of the canals. However, it is very toxic to the periapical tissues if it was extruded beyond the apex [8]. A new root canal irrigant, Biopure MTAD was reported to be effective as a final rinse to remove the smear layer with minimal erosive changes and has the ability to disinfect infected root canal. The suggested regimen of Biopure MTAD is initial irrigation of the canals by $(1.3 \%)$ sodium hypochlorite during instrumentation and final 5 minute irrigation with $5 \mathrm{ml}$ Biopure MTAD [9].

\section{Materials and Methods \\ Sensitivity test}

Two $\mathrm{ml}$ of each of the five tested materials mentioned in (Table 1) were prepared in each of the five $10 \mathrm{ml}$ sterile test tubes (Wassermann tubes). An overnight trypticase soy broth (Sparks, MD 21152 USA. 38800 Le Pont de Claix, France) culture of Enterococcus faecalis (ATCC 29212) were standardized to 0.4 optical densities and measured at $600 \mathrm{~nm}$. Six sterile glass plates of Mueller-Hinton agar (Sparks, MD 21152 USA. 38800 Le Pont de Claix, France) with 5\% defibrinated sheep blood were prepared. One hundred micro-liters of the broth were spread on the agar media for each of the six glass plates. Every glass plate was divided equally and marked by numbers for six parts according to the six tested solutions. 36 sterile filter disc, one-quarter inch in size (radius $6 \mathrm{~mm}$ ), were divided into six groups, six each, for each glass plate. Approximately $15 \mu \mathrm{l}$ of each prepared tested solutions were loaded on the corresponding disc and placed in its place according to its number for each glass plates. These procedures were repeated for the remaining five glass plates. The six plates were incubated at $37^{\circ} \mathrm{C}$ for 48 hours and the zones of inhibition were then measured in $\mathrm{mm}$. The data obtained were recorded and statistically analyzed.

\begin{tabular}{|l|c|c|c|}
\hline No. & Tested materials & Mean & S.D \\
\hline 1 & Biopure MTAD & 22.3 & \pm 3.26 \\
\hline 2 & $\begin{array}{c}1.3 \% \text { Sodium hypochlorite }+ \\
\text { Biopure MTAD (1ml to1ml) }\end{array}$ & 17.5 & $2.25 \pm$ \\
\hline 3 & $\begin{array}{c}\text { 20\% Hydrogen peroxide + } \\
\text { Biopure MTAD (1ml to1ml) }\end{array}$ & 20.3 & \pm 2.94 \\
\hline 4 & $\begin{array}{c}5.25 \% \text { Sodium hypochlorite } \\
\text { (full strength) }\end{array}$ & 7 & $1.10 \pm$ \\
\hline 5 & $\begin{array}{c}2.5 \% \text { Sodium hypochlorite } \\
\text { (diluted form) }\end{array}$ & 6 & 0.0 \\
\hline 6 & Ampicillin (antibiotic) & 16.5 & \pm 1.22 \\
\hline
\end{tabular}

Table 1: Means of the inhibition zone values for tested solutions in ( $\mathrm{mm})$.

Least Significant Difference $(\mathrm{LSD})=2.5 ; \mathrm{P}<0.0001$

Antimicrobial activity in contaminated root canals of extracted teeth

Selection and standardization of root canals

A total of 55 recently extracted sound permanent single-canaled human teeth were collected. The teeth were cleaned from any calculus deposits and soft tissue debris by ultrasonic scalar, and then stored in distilled water containing $2 \%$ sodium azide (as an antimicrobial agent) until use. Crowns of all the teeth were removed with a diamond disk and the root lengths were standardized to approximately $14 \mathrm{~mm}$. For standardization of root canal diameter, Gates-Glidden drills sizes 4, 3 and 2 were used to prepare the root canal orifices. The apical thirds were prepared with K-file sizes 25 , 30,35 and 40 using the balanced force technique. The canals were irrigated with $1 \mathrm{ml}$ of freshly prepared $1.3 \%$ sodium hypochlorite solution after each file used. All the root canals received a flush of 5 $\mathrm{ml}$ of $17 \%$ EDTA (pH 7.2) for 1 min to remove the smear layer. These procedures were followed by final irrigation with $5 \mathrm{ml}$ physiologic saline solution. Root canals were dried with sterile paper points. All the roots were sterilized by autoclaving $\left(15 \mathrm{~min}\right.$ at $\left.121^{\circ} \mathrm{C}\right)$. The roots were then kept in sterile water to avoid dehydration until use.

\section{Infection of root canals}

The antibacterial effect test of the used materials has been already done under a laminar flow hood in the lab of microbiology and immunology department at the faculty of Pharmacy in Suez Canal University. For infection of the root canals, an overnight trypticase soy broth inoculated with Enterococcus faecalis (ATCC 29212) were standardized to 0.4 optical density and measured at $600 \mathrm{~nm}$. The apical foramina of all the roots were sealed with soften wax. Freshly prepared sterile trypticase soy broth was distributed in sterilized Wassermann tubes (approximately 110 tubes) and each tube contained $1 \mathrm{ml}$ of the broth for culturing of the swabs. 
Five teeth were selected randomly to be cultured before infection of roots to act as the negative control group (-ve). Approximately $15 \mu \mathrm{l}$ of the sterile broth were introduced into each of these root canals. Two or three sterile paper points were introduced into every root canal, and then transferred aseptically into its markedly corresponding Wassermann tube for each root. The remaining fifty roots were arranged vertically in a suitable rack, and then approximately $15 \mu \mathrm{l}$ of the broth were introduced into each of the root canals and incubated for 24 hours. After that time, another dose of approximately $15 \mu \mathrm{l}$ of the broth was introduced into each of the roots and then incubated for another 24 hours. Another five roots were selected after the whole incubation period (48 hours) and cultured in a similar way of negative control to act as positive control group (+ve). The remaining forty-five roots were divided into three equal groups, 15 roots each, corresponding to the used irrigant solutions $\mathrm{M}, \mathrm{C}$ and $\mathrm{D}$.

\section{Disinfection test of infected roots}

For the first group, two or three sterile paper points were introduced into every infected root canal, and then transferred aseptically into markedly corresponding Wassermann tube for each root forming the first swabs. After that each root canal was irrigated with freshly prepared Bio pure MTAD (DENTSPLY/ Tulsa Dental, Tulsa, OK), $1 \mathrm{ml}$ as an initial rinse and soaked for 5 minutes. A final rinse was done with $4 \mathrm{ml}$ for each canal. Each root canal was dried with sterile paper points. Approximately $15 \mu$ of sterile trypticase soy broth were introduced into every root canal. Two or three sterile paper points were introduced into every root canal, and then moved aseptically into its markedly corresponding Wassermann tube for each root forming the second swabs. For the second group, two or three sterile paper points were introduced into every infected root canal, and then moved aseptically into markedly corresponding Wassermann tube for each root forming the first swabs. After that each root canal was irrigated with $5 \mathrm{ml}$ $5.25 \%$ sodium hypochlorite (full strength) (Sigma, Pool, UK.). Each root canal was dried with sterile paper points. Approximately 15 $\mu \mathrm{l}$ of sterile trypticase soy broth were introduced into every root canal. Two or three sterile paper points were introduced into every root canal and then moved aseptically into its markedly corresponding Wassermann tube for each root forming the second swabs. For the third group, two or three sterile paper points were introduced into every infected root canal, and then transferred aseptically into markedly corresponding Wassermann tube for each root forming the first swabs. After that each root canal was irrigated with $5 \mathrm{ml} 2.5 \%$ sodium hypochlorite (diluted form). Each root canal was dried with sterile paper points. Approximately 15 $\mu \mathrm{l}$ of sterile trypticase soy broth were introduced into every root canal. Two or three sterile paper points were introduced into every root canal, and then moved aseptically into its markedly corresponding Wassermann tube for each root forming the second swabs. All the marked Wassermann tubes were incubated at $37^{\circ} \mathrm{C}$ for 48 hours then microbial growth of Enterococcus faecalis was verified by turbidity of the broth. Turbid trypticase soy broth tubes were recorded positive (+ve) and the clear ones were recorded negative (-ve). The data obtained were recorded and statistically analyzed.

\section{Results}

Sensitivity test

ANOVA statistical analysis showed significant difference between the tested materials, after incubation at $37 \circ \mathrm{C}$ for 48 hours. Bio pure MTAD revealed the highest mean inhibition zone value $(22.3+3.26 \mathrm{~mm})$. But zones of inhibition of Bio pure MTAD decreased by using additives like (20\%) hydrogen peroxide and (1.3\%) sodium hypochlorite with no significant difference between them and with that recorded by Ampicillin. While, Bio pure MTAD, its additives and Ampicillin revealed highly significant differences with full strength sodium hypochlorite that has the lowest mean inhibition zone value and the diluted (2.5\%) sodium hypochlorite that did not record any inhibition zones, as shown in (Table 1) and (Figure 1)

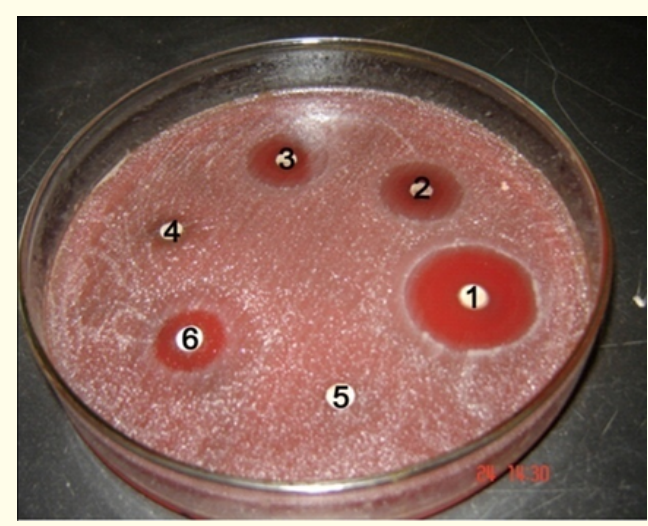

Figure 1: Sensitivity test of the tested materials.

\section{Antimicrobial activity in contaminated root canals of extracted} teeth

Chi-Square values mentioned after statistical analysis, for the negative control group, all the tubes were clear (100\% negative). While, the positive control group, all the tubes were turbid $(100 \%$ positive). All the tubes of Bio pure MTAD group were clear. For full strength $(25.5 \%)$ sodium hypochlorite group, many tubes were clear and some were turbid. The diluted sodium hypochlorite $(2.5 \%)$ group showed some tubes were clear and many were turbid as shown in (Table 2) and (Figure 2 to 4 ). 


\begin{tabular}{|c|c|c|}
\hline Groups & Negative & Positive \\
\hline Negative control & $100 \%$ & $0 \%$ \\
\hline Positive control & $0 \%$ & $100 \%$ \\
\hline Biopure MTAD & $100 \%$ & $0 \%$ \\
\hline $5.25 \%$ sodium hypochlorite (full strength) & $80.00 \%$ & $20 \%$ \\
\hline $2.5 \%$ sodium hypochlorite (diluted) & $40 \%$ & $60 \%$ \\
\hline
\end{tabular}

Table 2: Chi-Square values of antimicrobial activity test in contaminated root canals of extracted teeth. Chi-Square value $=26.9$;

$$
\mathrm{P}<0.0001
$$

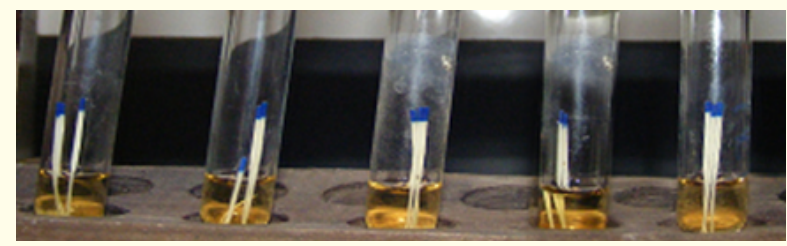

Figure 2: Clear trypticase soy broth tubes of Biopure MTAD group recording negative (-ve) results.

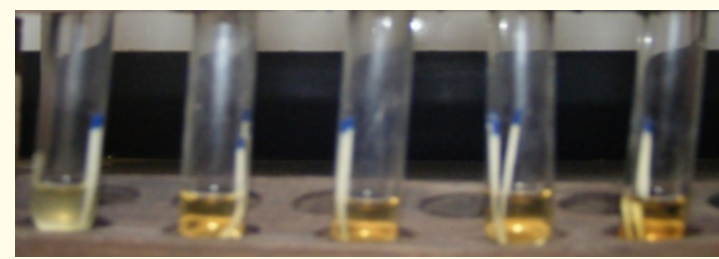

Figure 3: Clear and turbid trypticase soy broth tubes of 5.25\% sodium hypochlorite (full strength) recording negative $(-v e)$ and positive (+ve) results.

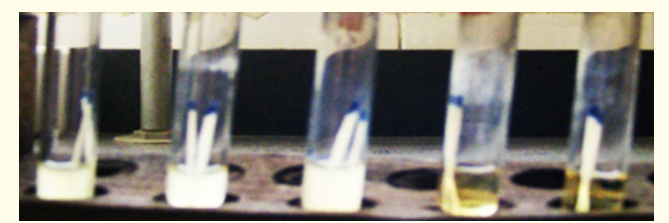

Figure 4: Clear and turbid trypticase soy broth tubes of 2.5\% sodium hypochlorite (diluted) recording negative (-ve) and positive (+ve) results.

\section{Discussion}

The sensitivity test, revealed that $(5.25 \%)$ and $(2.5 \%)$ sodium hypochlorite recorded nearly no effect against $E$. faecalis. Hassan., et al. [10] and Vianna and Gomes [11] revealed also that a small or no zone of growth inhibition of E. faecalis was observed. The sensitivity test revealed also that Bio pure MTAD recorded the greatest ability in inhibiting the growth of Enterococcus faecalis among all the tested materials. The antimicrobial efficiency of Bio pure MTAD was decreased by the addition of (20\%) hydrogen peroxide and (1.3\%) sodium hypochlorite. Johal., et al. [12] reported that large zone of inhibiting the growth of Enterococcus faecalis was observed with Bio pure MTAD compared to degradation products. Davis., et al. [13] investigated the antimicrobial action of Biopure MTAD, 2\% chlorhexidine and 5.25\% sodium hypochlorite ( $\mathrm{NaOCl}$ ) against Enterococcus faecalis using the sensitivity test. Bio pure MTAD showed the largest zones of microbial inhibition than $5.25 \%$ $\mathrm{NaOCl}$ and $2 \% \mathrm{CHX}$. The zone of inhibition between $\mathrm{NaOCl}$ and $\mathrm{CHX}$ was not significant. The, Biopure MTAD completely disinfected the roots and the teeth by inhibiting the growth of Enterococcus faecalis. Shabahang and Torabinejad [14] found that the grouping of $1.3 \% \mathrm{NaOCl}$ as a root canal irrigant and Bio pure MTAD as a final rinse was significantly more efficient against Enterococcus faecalis. Mohammadi and Shahriari [15] also stated that the Biopure MTAD group and sodium hypochlorite group exhibited the least and highest numbers of colony-forming units (CFUs). The antimicrobial efficiency of Biopure MTAD was significantly superior to that of sodium hypochlorite. Shenoi., et al. [16] also investigated Bio Pure MTAD had a significantly higher mean inhibition zone against $\mathrm{E}$ faecalis than the other irrigants. Furthermore, physical removal of Enterococcus faecalis through clean of the root canal remains essential, since remnants may tolerate the inflammation [17]. Charlie., et al. [18] MTAD has got higher antibacterial action against E. faecalis, than $2 \%$ chlorhexidine gluconate, 17\% EDTA, and 5\% NaOCl. Neglia., et al. [19] and Estrela., et al. [20] emphasized that the sodium hypochlorite has low antimicrobial efficiency against Enterococcus faecalis. Moreover, Tirali., et al. [21] added that increasing sodium hypochlorite concentration increases its antimicrobial action. The results of our study was in disagreement with other researchers [22-24] who recorded that sodium hypochlorite was superior in its antimicrobial abilities compared with other tested materials.

\section{Conclusions}

Biopure MTAD recorded the best results in respect of inhibiting the growth of Enterococcus faecalis. Moreover, additives as (1.3\%) sodium hypochlorite and (20\%) hydrogen peroxide decreased its efficiency against Enterococcus faecalis. Coupling the advantage of Bio pure MTAD and sodium hypochlorite as irrigating solutions during cleaning and shaping of endodontic treated teeth by using sodium hypochlorite during cleaning and shaping and Bio pure MTAD as a final rinse is beneficial.

\section{Bibliography}

1. Siqueira JF., et al. "Actinomyces species, Streptococci, and Enterococcus faecalis in primary root canal infections". Journal of Endodontics 28 (2002): 168-172.

2. Sundqvist G. "Associations between microbial species in dental root canal infections". Oral Microbiology and Immunology 7 (1992): 257-262. 
3. Nair PN and Schroeder HE. "Periapical actinomycosis". Journal of Endodontics 12 (1984): 567-570.

4. Kishen A., et al. "Chair side sensor for rapid monitoring of Enterococcus faecalis activity". Journal of Endodontics 30 (2004): 872-875.

5. Sundqvist G., et al. "Microbiologic analysis of teeth with failed endodontic treatment and the outcome of conservative retreatment". Oral Surgery, Oral Medicine, Oral Pathology, and Oral Radiology 85 (1998): 86-93.

6. Haffajee AD., et al. "Clinical and microbiological features of subjects with adult periodontitis who responded poorly to scaling and root planning". Journal of Clinical Periodontology 24 (1997): 767-776.

7. Nagayoshi M., et al. "Antimicrobial effect of ozonated water on bacteria invading dentinal tubules". Journal of Endodontics 30 (2004): 778- 781.

8. Dang E. "Comparison of sodium hypochlorite and Chlorhexidine Gluoconate: Quality of current evidence". Journal Youn Investment 19 (2008): 6-11.

9. Giardino L., et al. "Comparative evaluation of antimicrobial efficacy of sodium hypochlorite, MTAD, and Tetraclean against Enterococcus faecalis biofilm". Journal of Endodontics 33 (2007): 852-855.

10. Hassan HY., et al. "Evaluation of antimicrobial efficacy of four tested solutions against Enterococcus faecalis, an In vitro study". The Medical Journal of Cairo University 85.8 (2017): 3107-3112.

11. Vianna ME and Gomes BP. "Efficacy of sodium hypochlorite combined with chlorhexidine against Enterococcus faecalis In vitro". Oral Surgery, Oral Medicine, Oral Pathology, and Oral Radiology 107 (2009): 585-589.

12. Johal S., et al. "Comparison of the antimicrobial efficacy of $1.3 \% \mathrm{NaOCl} / \mathrm{BioPure}$ MTAD to $5.25 \% \mathrm{NaOCl} / 15 \%$ EDTA for root canal irrigation". Journal of Endodontics 33.1 (2007): 4851.

13. Davis JM., et al. "An In vitro comparison of the antimicrobial effects of various endodontic medicaments on Enterococcus faecalis". Journal of Endodontics 33 (2007): 567-569.

14. Shabahang S and Torabinejad M. "Effect of MTAD on Enterococcus faecalis - contaminated root canals of extracted human teeth". Journal of Endodontics 29 (2003): 576-579.

15. Mohammadi Z and Shahriari S. "Residual antibacterial activity of chlorhexidine and MTAD in human root dentin In vitro". Journal of Oral Science 50 (2008): 63-67.

16. Shenoi PR., et al. "In vitro evaluation of the antimicrobial efficacy of chitosan and other endodontic irrigants against Enterococcus faecalis". General Dentistry 64 (2016): 60-63.
17. Torabinejad M., et al. "The antimicrobial effect of MTAD: An In vitro investigation". Journal of Endodontics 29 (2003): 400-403.

18. Charlie KM., et al. "A Scanning electron microscope evaluation of smear layer removal and antimicrobial action of mixture of tetracycline, acid and detergent, sodium hypochlorite, Ethylenediaminetetraacetic acid, and Chlorhexidine Gluconate: an In vitro study". Journal of International Society of Preventive and Community Dentistry 8 (2018): 62-69.

19. Neglia R., et al. "Comparative in vitro and ex vivo studies on the bactericidal activity of Tetraclean, a new generation endodontic irrigant, and sodium hypochlorite". New Microbiologica 31 (2008): 57-65.

20. Estrela C., et al. "Efficacy of sodium hypochlorite and chlorhexidine against Enterococcus faecalis". Journal of Oral Science 16 (2008): 364-368.

21. Tirali RE., et al. "In vitro antimicrobial activity of several concentrations of $\mathrm{NaOCl}$ and Octenisept in elimination of endodontic pathogens". Oral Surgery, Oral Medicine, Oral Pathology, and Oral Radiology 108 (2009): 117-120.

22. Stoll R., et al. "The disinfecting effect of ozonized oxygen in an infected root canal: an In vitro study". Quintessence International 39 (2008): 231-236.

23. Meire MA., et al. "Effectiveness of different laser systems to kill Enterococcus faecalis in aqueous suspension and in an infected tooth model". International Endodontic Journal 42 (2009): 351-359.

24. Kuştarci A., et al. "Bactericidal effect of KTP laser irradiation against Enterococcus faecalis compared with gaseous ozone: an ex vivo study". Oral Surgery, Oral Medicine, Oral Pathology, and Oral Radiology 107 (2009): 73-79.

\section{Assets from publication with us}

- Prompt Acknowledgement after receiving the article

- Thorough Double blinded peer review

- Rapid Publication

- Issue of Publication Certificate

- High visibility of your Published work

Website: www.actascientific.com/

Submit Article: www.actascientific.com/submission.php Email us: editor@actascientific.com

Contact us: +919182824667 